\title{
Polyhydroxybutyrate: A Useful Product of Chlorotic Cyanobacteria
}

\author{
Moritz Koch Karl Forchhammer \\ Interfaculty Institute of Microbiology and Infection Medicine Tübingen, Eberhard Karls University Tübingen, \\ Tübingen, Germany
}

\section{Keywords}

Polyhydroxybutyrate $\cdot$ Bacteria $\cdot$ Physiology $\cdot$ Cyanobacteria $\cdot$ Survival strategies

\section{Abstract}

Polyhydroxybutyrate (PHB) is a carbon polymer with diverse functions, varying greatly on the organism producing it. This microreview describes the current knowledge about PHB metabolism, structure, and different physiological roles with a special focus on cyanobacteria. Despite the physiological function of PHB in the cyanobacterial phylum still being unknown, these organisms provide the unique opportunity to directly convert atmospheric $\mathrm{CO}_{2}$ into bioplastic using a solar-based process. Recent research on PHB metabolism in the cyanobacterial model organism Synechocystis revealed a sophisticated control of PHB granule formation. Novel insights about the metabolic background of PHB synthesis resulted in the engineering of the first cyanobacterial superproducer strain.

C 2021 The Author(s)

Published by S. Karger AG, Basel

\section{The Characteristics and Classification of Polyhydroxyalkanoates}

Polyhydroxybutyrate (PHB) was discovered in Bacillus megaterium in 1926 [Lemoigne, 1926]. PHB is composed of 3-hydroxy-butyrate monomers. When several 3-hydroxybutyrate monomers are connected via an ester bond, they form PHB. The latter accumulates in the form of water-insoluble inclusions within the cell. Six decades after $\mathrm{PHB}$ was first discovered, in 1983 researchers showed that when grown on octane, Pseudomonas oleovorans produces poly-beta-hydroxyoctanoate granules [de Smet et al., 1983]. This was the first time that the microbial production of other polyhydroxyalkanoates (PHA) was discovered. PHAs are classified in three different groups, depending on the length of the monomer: short- $\left(\mathrm{C}_{3}-\mathrm{C}_{5}\right)$, medium- $\left(\mathrm{C}_{6}-\mathrm{C}_{16}\right)$ and long-chain-length PHA (scl, mcl, and lcl, respectively) [Reddy et al., 2003]. A selection of different PHA types is given in Figure 1.

Production of PHA is described from representatives from all three kingdoms of life: archaea, bacteria, and eukaryotes. Just recently it has been reported that also eukaryotic algae can naturally produce $\mathrm{PHB}$, namely Chlorella [Cassuriaga et al., 2018] and Botryococcus

Correspondence to:

Karl Forchhammer, karl.forchhammer@uni-tuebingen.de karger@karger.com www.karger.com/mip

Karger $\stackrel{\text { ' }}{=}$

BOPEN ACCESS
(C) 2021 The Author(s).

Published by S. Karger AG, Basel

This is an Open Access article licensed under the Creative Common Attribution-NonCommercial-4.0 International License (CC BY-NC) (http://www.karger.com/Services/OpenAccessLicense), applicable to the online version of the article only. Usage and distribution for commercial purposes requires written permission. 


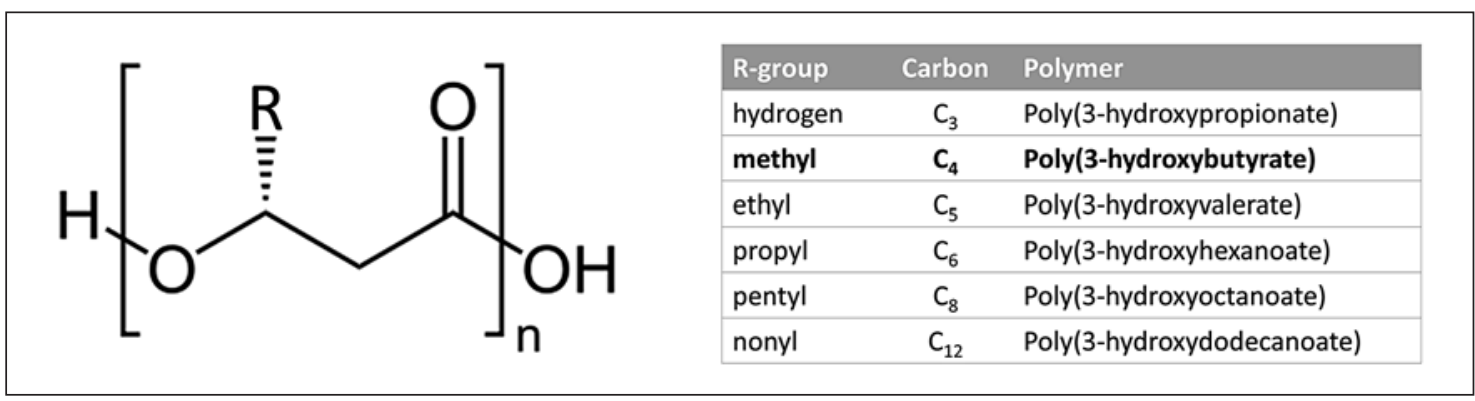

Fig. 1. General structure of PHA. Depending on the side chain R, several different types of PHA are formed. In the case of PHB (bold), the side chain corresponds to a methyl group. The monomeric units (n) of PHAs are linked via an ester bond between the hydroxy- and the carboxyl groups, forming the respective PHA.

Fig. 2. $\mathrm{PHB}$ synthesis pathway. Two units of acetyl-CoA are condensed to acetoacetyl-CoA via PhaA. Next, $\mathrm{PhaB}$ reduces it to 3-hydroxybutyryl-CoA. The latter serves as a monomer, which gets polymerized to $\mathrm{PHB}$ by PhaC or PhaEC, depending on the organism.

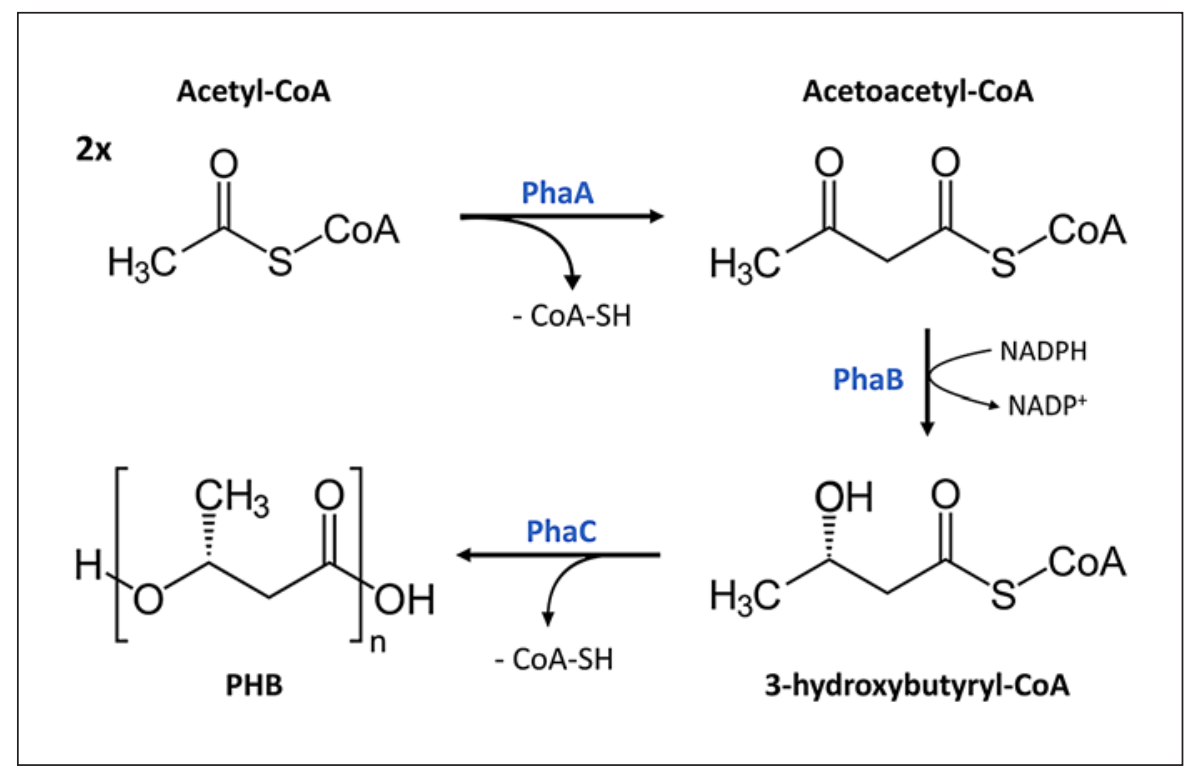

[Kavitha et al., 2016]. However, PHA production is best characterized in heterotrophic bacteria, such as $P$. putida [Timm and Steinbüchel, 1990], Cupriavidus necator (formerly known as Ralstonia eutropha) [Yabuuchi et al., 1995], B. megaterium [Griebel et al., 1968], Rhodococcus ruber [Haywood et al., 1991] or Acinetobatcer sp. [Schembri et al., 1995]. Since PHB is by far the most widely distributed type of PHA in bacteria, this review focuses mostly on PHB. For the sake of completeness, however, some aspects related to other PHAs are also mentioned.

Due to its physical and chemical properties, PHB is often considered as a potential substitute for thermoplastic polymers, such as polypropylene. However, there are important differences compared to commonly used plas- tics. For example, PHB exhibits low elasticity but high rigidness, which are undesirable material properties for many applications [Van der Walle et al., 2001].

\section{PHB Metabolism}

PHB synthesis starts with acetyl-CoA monomers. In the first step, the enzyme PhaA, an acetyl-CoA acetyltransferase (also termed acetoacetyl-CoA thiolase) catalyzes the condensation of two units of acetyl-CoA to acetoacetyl-CoA. In the next step, an acetoacetyl-CoA reducatase (PhaB), reduces acetoacetyl-CoA to 3-hydroxyacetly-CoA, while oxidizing one molecule of $\mathrm{NADPH}$ to $\mathrm{NADP}^{+}$. Finally, a synthase connects a 3-hydroxyacetly-CoA to an elongated $\mathrm{PHB}$ poly- 


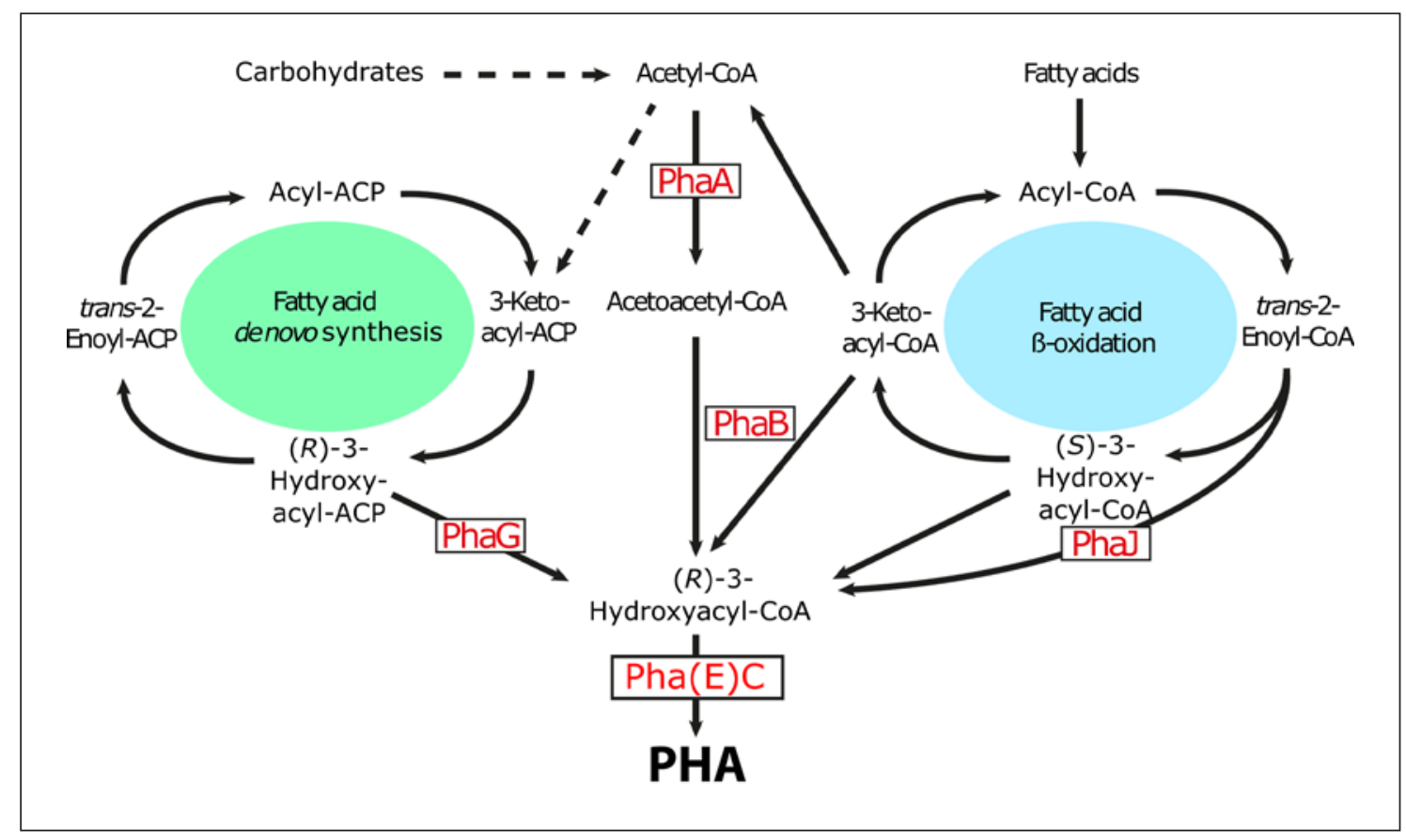

Fig. 3. PHA metabolism and different metabolic pathways [Koch and Forchhammer, 2020]. Important enzymes are highlighted in red. Depending on the organism, the PHA synthase is either PhaC or PhaEC. Not shown is the PHA depolymerization, which is catalyzed by PhaZ.

mer (Fig. 2). In cyanobacteria, this synthase consists of two different dimers, namely $\mathrm{PhaE}$ and $\mathrm{PhaC}$, making it a member of class III PHA synthases [Hein et al., 1998].

The PHB polymer can be catabolized by PHA depolymerases, termed PhaZ. This can take place either intra- or extracellularly, depending on their purpose. In C. necator, there are seven different depolymerases annotated, indicating the importance of a regulated mobilization of PHB [Pohlmann et al., 2006]. PhaZ degrades PHB into monomers of crotonylCoA [Eggers and Steinbüchel, 2013], which is then further metabolized in the $\beta$-oxidation pathway. It was furthermore shown that the phasins PhaP have a strong impact on the degradation of PHB [Eggers and Steinbüchel, 2014].

Besides the enzymes mentioned above, some species, like $P$. putida, possess further enzymes, which enable them to convert additional substrates into PHA. One enzyme is PhaJ, which converts enoyl-CoA into R-3-hydroxyacyl-CoA and thereby connects the fatty acid $\beta$-oxidation with the PHA synthesis [Liu et al., 2016]. PhaG, another enzyme found in P. putida, converts acylACP to (R)-3-acyl-CoA and thereby links the fatty acid de novo synthesis to the PHA metabolism. The fatty acid and PHA pathways are further interconnected by enzymes, which catalyze reactions in both pathways. For ex- ample, the enzyme FabG, which is actually part of the fatty acid biosynthesis, can also catalyze the same function as PhaB, but with lower catalytic efficiency [Zhang et al., 2017]. An overview of the most important pathways involved in the PHA metabolism is shown in Figure 3 (except the PHA depolymerization). Further details can be found in recent reviews [Zhang et al., 2020].

With all those enzymes in place, the PHA cycle is complete and acetyl-CoA can be metabolized to PHA and back again. Interestingly, some studies suggest that there could be a constant carbon flow around PHA synthesis and degradation, creating a potential futile cycle [Ren et al., 2009; de Eugenio et al., 2010]. Thereby the cells could balance metabolic intermediates and adapt the cell to changing environmental conditions. In support of this hypothesis, PHA synthase and depolymerase were shown to be simultaneously active in P. putida [Arias et al., 2013].

\section{Proteins Associated with PHB Granules}

Despite the apparent simplicity of $\mathrm{PHB}$, the polymer is organized in highly organized structures with a spherical shape, called PHB granules. Due to this complexity, the 
granules are also named carbonosomes to highlight that PHB granules are rather subcellular organelles and not just a prolonged chain of molecules [Jendrossek, 2009]. A set of different proteins, termed as granule-associated proteins (GAP), are located on the granule-surface. They serve various different purposes, such as structural, biosynthetic, catabolic, and regulatory functions.

A specific group are phasins, which are low-molecular-weight proteins attached to the surface of PHB granules [Mezzina and Pettinari, 2016]. They are very abundant and shield the hydrophobic PHB surface from the hydrophilic cytoplasm, as well as prevent the interaction with other proteins. A representative of such phasins is PhaP1 from C. necator, the most important phasin in this species [Pötter et al., 2004; Pfeiffer and Jendrossek, 2012]. The amount of PhaP1 is tightly regulated and corresponds with the amount of intracellular PHB [York et al., 2001]. In P. putida, PhaF and PhaI are the main GAP [Prieto et al., 1999].

Besides just covering the granules surface, GAP exhibit further functions. For example, PhaM in C. necator, which binds to $\mathrm{PhaC}$ as well as chromosomal DNA, ensures equal distribution among the daughter cells. Additionally, PhaM activates the $\mathrm{PHB}$ synthase $\mathrm{PhaC} 1$ and thereby directly influences the PHB metabolism [Pfeiffer and Jendrossek, 2014]. The regulator PhaR regulates pha genes on a transcriptional level [Pötter et al., 2002; York et al., 2002]. It was long disputed whether PHB granules are covered with a phospholipid membrane. During PHB preparations, phospholipids have often been found in vitro. However, using a phospholipid-specific fluorescent reporter, Bresan et al. [2016] could eventually demonstrate that $\mathrm{PHB}$ granules do not contain phospholipids on their surface in vivo. This demonstrates that phospholipids are merely contaminations of the preparation procedure.

\section{Physiological Role of PHB in Bacteria}

The ability to produce PHB is widespread in the bacterial kingdom. A phylogenetic analysis found the presence of the phaC gene in species from 40 different genera and within a wide range of taxonomic groups, highlighting how abundant the ability to produce PHB is [Kalia et al., 2007]. PHBs are in general considered as storage molecules of carbon and energy, which are built up in periods of carbon excess to be used in times of carbon shortage [Anderson and Dawes, 1990]. This long-time belief still holds true for many bacteria, although more and more other physiological functions have recently been discovered. Also, for some bacteria like cyanobacteria, no physiological relevance of PHB was yet discovered. The most important roles of PHB are described below.

In many organisms, $\mathrm{PHB}$ accumulates under conditions of nutrient limitation or unbalanced conditions, such as nitrogen limitation [Anderson and Dawes, 1990]. However, there are also species, like $C$. necator, which do accumulate $\mathrm{PHB}$ even during normal growth and under balanced conditions [Jendrossek and Pfeiffer, 2014]. Nevertheless, the amount of accumulated PHB is usually higher when grown under nutrient limitation. Besides serving as a storage polymer, $\mathrm{PHB}$ has been suggested to increase resistance against various kinds of stresses. In general, 3-hydroxybutyrate oligomers can protect bacteria against hydroxyl-radicals [Koskimäki et al., 2016]. In Azospirillum brasilense for example, PHB-deficient strains are more susceptible against abiotic stresses, such as UV irradiation, heat, desiccation, osmotic pressure, and osmotic shock [Kadouri et al., 2003a]. In Sinorhizobium strains, an induced PHB accumulation after exposure to high salt concentrations was observed [Arora et al., 2006]. In Aeromonas hydrophila the production of $[\mathrm{P}(3 \mathrm{HB}-\mathrm{co}-3 \mathrm{HHx})]$ copolymers results in increased resistance against a wide variety of abiotic stresses, including UV irradiation, hydrogen-peroxide, ethanol, heat and cold treatments, and high osmotic pressure [Zhao et al., 2007]. Interestingly, a study investigating $P$. oleovorans showed that even the deletion of the PHA depolymerase PhaZ was sufficient to increase the sensitivity towards hydrogen-peroxide and heat shock [Ruiz et al., 2004]. This demonstrates that the entire PHB metabolism, including its mobilization, is important for providing stress tolerance to the PHB-producing cells, rather than the sheer presence of the polymer [Castro-Sowinski et al., 2009]. PHB was furthermore shown to increase the number of viable cells by protecting $C$. necator cells against cold stress [Nowroth et al., 2016]. Additionally, in Herbaspirillum seropedicae, studies demonstrated that PHB reduces intracellular redox stress, potentially by eliminating a surplus of reducing equivalents and thereby serving as an electron sink [Batista et al., 2018]. Chromatium vinosum, an anoxygenic phototrophic bacterium, converts glycogen to PHB under dark, anaerobic conditions. This provides the strain with the advantage over other fermenting organisms, which commonly secrete their fermentation products and thereby lose carbon [Van Gemerden, 1968]. Another role of PHB can be found in B. cereus: this bacterium produces most $\mathrm{PHB}$ just before the formation of spores and degrades it during spore germination, indicat- 
ing the importance of $\mathrm{PHB}$ for surviving their dormant state [Valappil et al., 2007; Castro-Sowinski et al., 2009]. Several studies also found a connection between PHB production and the formation of EPS (exopolysaccharides), since impaired PHB production in some cases resulted in a higher EPS production [Wang et al., 2008], while with a lower EPS production in other strains [Kadouri et al., 2003b]. When the EPS production was higher, the authors suggested that the increased EPS production is attributable to the intracellular mobilization of carbon sources (PHB).

Interestingly, $\mathrm{PHB}$ producing $A$. brasilense was able to endure long periods of starvation, but it also showed certain disadvantages compared to a PHB-free mutant, such as lower motility or impaired root adhesion and EPS production [Kadouri et al., 2002]. This indicates that PHB formation is not always an advantage, but depends on the environmental conditions. Similarly, Sinorhizobium bacteria were shown to use a bet-hedging strategy to produce offspring with higher or lower amounts of PHB, adapted for long- or short-period starvation, respectively [Ratcliff and Denison, 2010]. Another interesting function of PHB could be the provision of nutrients to a microbial community [Prieto et al., 2016]. When a PHB producer is lysed, for example by predatory bacteria, it releases its PHB granules to the environment [Jendrossek and Handrick, 2002]. Thereby, the energy-rich carbon polymer is made available to the microbial community [Prieto et al., 2016]. In line with this, predatory Bdellovibrio cells have a growth advantage when preying on PHA-producing cells compared to PHB-free mutants [Martínez et al., 2013]. For more details on the physiological functions of PHB in bacteria, readers are referred to recent reviews [Castro-Sowinski et al., 2009; Müller-Santos et al., 2020; Obruca et al., 2020].

\section{PHB in Cyanobacteria}

Cyanobacteria comprise a large group of PHB-producing bacteria. They grow photoautotrophically and are the only bacteria that perform oxygenic photosynthesis. This allows cyanobacteria to occupy almost all illuminated habitats. In a recent study, 137 different cyanobacterial strains were investigated for their ability to produce PHB. Out of these, 134 were PHB producers [KaewbaiNgam et al., 2016]. Interestingly, a phylogenetic analysis revealed that the full set of functional phaABC genes presumably evolved first in cyanobacteria, indicating its importance for cyanobacterial growth [Kalia et al., 2007].
Despite that, its physiological function is yet undiscovered. In recent years, this question was addressed by comparing a PHB-free $\triangle$ phaEC strain to a PHB-producing wild-type, but due to the absence of a clear phenotype, the function of PHB remains puzzling [Damrow et al., 2016; Koch et al., 2020a]. Cyanobacteria produce PHB mostly under unbalanced growth conditions, for example when they are grown in a medium lacking either nitrogen, phosphate, or potassium [Kaewbai-Ngam et al., 2016]. Additional organic carbon sources, like acetate or fructose, can further increase the PHB production [Panda et al., 2006]. The PHB metabolism in cyanobacteria is best described during nitrogen starvation, which triggers a process called chlorosis [Klotz et al., 2016; Doello et al., 2018]. PHB slowly accumulates during the course of several weeks; depending on the cyanobacterial strain, up to $25 \%$ PHB per cell-dry-weight can be accumulated [Kaewbai-Ngam et al., 2016]. PHB is commonly stored in a few granules, which are located in the middle of the cell [Hauf et al., 2015]. Compared to other PHB producers, only relatively little is known about the proteins which are involved in PHB metabolism. Although two new proteins were recently found to play a role (the phasin $\mathrm{PhaP}$ as well as the regulator Slr0058) [Hauf et al., 2015; Koch et al., 2020c], more proteins, such as PHB-degrading enzymes, are to be discovered. Most studies were performed in the strain Synechocystis sp. PCC 6803 (hereafter Synechocystis), which serves as a well-characterized model strain for cyanobacterial metabolism. In the following, the latest discoveries and hints towards PHB's physiological role are summarized.

\section{The Role of PHB during Nutrient Limitation}

Cyanobacteria are known for producing, in addition to $\mathrm{PHB}$, a variety of different storage compounds, such as glycogen, cyanophycin, or polyphosphate. In contrast to the unknown function of $\mathrm{PHB}$, the polymers glycogen and cyanophycin can clearly be linked to carbon and nitrogen storage metabolism, respectively [Doello et al., 2018; Watzer and Forchhammer, 2018]. As previous studies have shown, glycogen is the main carbon and energy storage compound under conditions of nutrient limitation [Klotz et al., 2016; Doello et al., 2018] and its biosynthetic genes were found in all cyanobacterial genomes [Beck et al., 2012]. It is remarkable that cyanobacteria produce two different carbon polymers, since most other microorganisms produce just one. This clearly indicates that the two carbon polymers have different functions. Glycogen synthesis is extremely dynamic and is immediately induced when cells experience nitrogen limitation. 
Since glycogen synthesis mutants are unable to survive nitrogen chlorosis, glycogen metabolism appears to be pivotal for the acclimation to nitrogen deprivation [Klotz and Forchhammer, 2017; Doello et al., 2018]. Mutants that are deficient in the major glycogen-degrading phosphorylase GlgP2 do not accumulate PHB during chlorosis. From these analyses, it could be concluded that glycogen is slowly catabolized and converted into PHB during prolonged nitrogen starvation [Koch et al., 2019]. The cyanobacterium Synechocystis PCC 6803 operates at least three parallel metabolic routes to catabolize glycogen: the oxidative pentose phosphate (OPP) pathway, the EntnerDoudoroff (ED) pathway, and the Emden-MeyerhofParnas (EMP) pathway [Chen et al., 2016]. Analysis of mutants in which one or two of these pathways were disrupted showed that mutants in the ED and OPP pathways accumulated similar amounts of PHB as the wild-type, whereas mutants with a defect EMP pathway converted much less glycogen into PHB [Koch et al., 2019]. This effect was even more pronounced when the cells were cultivated under light/dark regime [Koch et al., 2020a]. Although Synechocystis normally accumulates more PHB under this condition, a mutant, which is unable to use the EMP pathway, produces almost no PHB. In contrast, the wild-type and a mutant unable to use either the ED or the OPP pathway $(\Delta z w f)$, produced approximately $15 \% \mathrm{PHB}$ per cell dry weight.

Acetyl-CoA, a key glycolytic product, serves as the precursor for the subsequent PHB synthesis, while the formation of glycogen requires phosphorylated glucose residues. This further suggests a role of glycogen as a quickresponse energy and carbon storage, since glucose-1P can directly be metabolized via any of the three pathways (ED, OPP, EMP) to provide energy for the cells' anabolism. In contrast, acetyl-CoA rather serves as a building block for various anabolic reactions (e.g., in lipid metabolism) and anaplerotic reactions related to the TCA cycle. While glycogen quickly accumulates or degrades within just 2 days at the beginning or end of nitrogen chlorosis, respectively, the formation of PHB takes much longer, lasting for several weeks after the induction of nitrogen starvation [Koch et al., 2020c].

Remarkable differences between PHB and glycogen are that glycogen shows a higher solubility in water than PHB, and that PHB forms much larger granules than glycogen. It was hypothesized that the relatively small glycogen granules could serve as a quick-response carbon storage due their easy accessibility based on a larger surfaceto-volume ratio, whereas the large $\mathrm{PHB}$ granules could instead form a long-term storage. However, under condi- tions of nitrogen limitation, no physiological differences were ever discovered between wild-type and a PHB-free $\triangle p h a E C$ mutant strain, even after prolonged starvation [Damrow et al., 2016; Klotz et al., 2016]. To test whether $\mathrm{PHB}$ can play a role in stress resistance, a recent study applied more than 30 different stresses to nitrogen-starved Synechocystis cells, including all of those stresses which are known for being relevant in other PHB-producing bacteria [Koch et al., 2020a]. However, the ability to produce PHB did not give the wild-type cells a growth advantage compared to a $\triangle p h a E C$ mutant in any of the tested conditions.

To better understand the metabolic role of PHB in Synechocystis, a recent study attempted to identify the so far unknown PHB depolymerase [Koch et al., 2020c]. Sequence homology analysis indicated that the gene slr0060 encodes for a putative esterase with a patatin-like domain, which is typical for some intracellular PHB depolymerases. The respective deletion mutant $\Delta$ slr0060 produced slightly less PHB than a wild-type control, but showed no further distinctive phenotype. Interestingly, the same study revealed that when a source of combined nitrogen was added to chlorotic cells to induce resuscitation to vegetatively growing cells, no net degradation of PHB in the cyanobacterial culture took place: the overall amount of PHB within a culture remained constant for several days [Koch et al., 2020c]. Only through cell growth and division, the PHB level per cell decreased gradually. However, fluorescence-microscopic analysis investigating Nile red-stained PHB revealed that the granules disaggregated and were spread among dividing daughter cells. The physiological function of this behavior and why PHB does not become actively metabolized has yet to be explained. Possibly cultivation in laboratory conditions did not trigger the degradation of $\mathrm{PHB}$, and the required environmental conditions which initiate $\mathrm{PHB}$ catabolism are still to be found. Therefore, to date it remains open whether cyanobacteria contain an intracellular PHB depolymerase as described for many other bacteria.

\section{Potential Role of PHB in Controlling the Redox State}

Besides conditions of nutrient limitation, glycogen is the essential energy and carbon storage during dark phases, where no photosynthesis can take place. Excess of carbon gets stored in the form of glycogen during the day, while during the night it releases its energy and carbon to sustain the cyanobacterial metabolism. Although no studies so far have shown a PHB accumulation during conditions of balanced growth, there are several indicators that PHB might play a role during dark phases. Tran-
72

Microb Physiol 2021;31:67-77 DOI: $10.1159 / 000515617$
Koch/Forchhammer 
scriptomic data from several studies have shown very clearly that all PHB-related genes are strongly up-regulated at the beginning of the night and down-regulated during the day [Kucho et al., 2005; Saha et al., 2016]. It was furthermore shown that PHB-related genes are strictly controlled by the circadian clock [Köbler et al., 2018]. Interestingly, the transcripts for PHB synthesis are counter-cyclically regulated to those of the glycogen metabolism, which are up-regulated during the beginning of the day and down-regulated during the beginning of the night. This hints towards a direct connection of both polymers, as it was already shown during nitrogen starvation [Koch et al., 2019]. However, also during diurnal (day/night) cultivation, a PHB-free mutant showed no growth phenotype, while growth of glycogen-free mutants was severely impaired [Damrow et al., 2016]. Furthermore, under conditions of nitrogen limitation, diurnal cultivation resulted in higher PHB levels than continuous light cultivation [Koch et al., 2020a]. This further highlights the relevance of dark phases for the formation of PHB.

PHB was previously suggested to play a role as a potential electron sink since each PHB subunit consumes one NADPH equivalent [De Philippis et al., 1992]. This hypothesis was further corroborated by studying a Synechocystis mutant in the gene sll0783 [Schlebusch and Forchhammer, 2010; Hauf et al., 2013]. The sll0783 gene belongs to the most strongly induced genes during nitrogen starvation and is the first gene of the Nit1C operon, a highly conversed gene cluster present in cyanobacteria and many other bacterial species (including proteobacteria and actinobacteria), enabling the utilization of cyanide [Jones et al., 2018]. Many heterotrophic bacteria possessing this gene cluster are able to produce PHB and to fix nitrogen. Since the entire operon was highly up-regulated in Synechocystis during nitrogen starvation, a role during nitrogen-chlorosis was suspected. Further analysis revealed that the $\Delta$ sllo783 mutant was unable to accumulate PHB due to a strongly decreased PHB synthase activity [Schlebusch and Forchhammer, 2010]. The inability of the $\Delta$ sll0783 mutant to sustain PHB synthesis could finally be attributed to the redox state of the cells: during nitrogen starvation, the $\mathrm{NADPH} / \mathrm{NADP}^{+}$ratio steadily increased in wild-type cells, whereas in the $\Delta$ sllo783 mutant it remained constant. Various treatments of the mutant cells that artificially increased the NADPH/NADP ${ }^{+}$ ratio restored $\mathrm{PHB}$ synthase activity and $\mathrm{PHB}$ synthesis [Hauf et al., 2013]. This highlights that the increased levels of NADPH during nitrogen starvation are an important trigger for $\mathrm{PHB}$ synthesis.

PHB in Cyanobacteria
According to the abovementioned facts, the formation of PHB would be particularly beneficial whenever a surplus of NADPH cannot be metabolized, for example because respiration is not possible and anaerobic processes are required. In this scenario, the formation of a PHB precursor would serve as an intracellular electron sink with the advantage to sustain all intracellular carbon, instead of secreting it like other fermentation products, such as acetate. As mentioned above, the EMP pathway plays a crucial role for the formation of $\mathrm{PHB}$. This pathway produces a lower NADPH/ATP ratio compared to the OPP or ED pathway. This is advantageous under conditions of $\mathrm{NADPH}$ excess, such as anaerobic growth. A previous study has shown that the EMP pathway is also the most relevant during fermentation processes in cyanobacteria [Stal and Moezelaar, 1997].

Another role of PHB could be a strategy to prevent metabolic spillover: a PHB-free C. necator mutant secretes pyruvate into its medium [Raberg et al., 2014]. A similar metabolic spillover was shown in Synechocystis mutants that are unable to synthesize glycogen and secrete pyruvate and 2-oxoglutarate under conditions of nitrogen starvation [Grundel et al., 2012]. Potentially, PHB could serve as an additional buffer for storing excess carbon, particularly because it is metabolically closer to pyruvate than glycogen. However, in contrast to the glycogen-deficient mutants, no studies have so far analyzed secreted organic acids in the supernatant of a PHB-free mutant. To further investigate this, future studies should analyze secreted metabolites under different growth conditions in a $\triangle p h a E C$ mutant and compare it to a wildtype.

\section{Cell Biology of PHB in Cyanobacteria}

The synthesis of PHB granules is tightly controlled. Based on sequence homology to other PHB-producing bacteria, several genes were identified which encode for proteins that are putatively involved in the PHB metabolism. One of those genes is $s l r 0058$, which encodes for a protein that contains a domain that is similarly found in PhaF from P. putida [Koch et al., 2020c]. Unlike the wildtype, a $\Delta$ slr0058 mutant produces small amounts of $\mathrm{PHB}$ during vegetative growth and shows delayed growth. This growth deficiency was clearly coupled to the presence of $\mathrm{PHB}$, since a phaEC knockout in the $\Delta$ slr0058 background recovered wild-type growth. The Slr0058 protein was furthermore shown to regulate the number of PHB granules within the cell. While a wild-type possesses around 3 granules per cell on average, the $\Delta$ slr0058 mutant contained about twice as many, but smaller gran- 
Fig. 4. Schematic overview of the current view of PHB metabolism in Synechocystis. After the induction of nitrogen starvation, cells fix inorganic $\mathrm{CO}_{2}$ and store it in the form of glycogen. Throughout the course of chlorosis, glycogen is catabolized, mainly via the EMP pathway, to form acetylCoA, the building block of PHB. Next, PhaA and PhaB convert acetyl-CoA subunits to 3-hydroxybutyryl-CoA at the expense of one NADPH molecule, which becomes oxidized to $\mathrm{NADP}^{+}$. Slr0058 initiates the formation of $\mathrm{PHB}$ granules and detaches as the PHB granules mature. The elongation of 3-hydroxybutyryl-CoA to the polymer PHB is catalyzed by the enzyme PhaEC, while PhaP shields the surface of the $\mathrm{PHB}$ granule. The color change indicates the progression of chlorosis, from vegetative growth to fully chlorotic (green to yellow, respectively). The process starts with the initial formation of glycogen, which eventually results in the accumulation of $\mathrm{PHB}$.

Fig. 5. Microscopic analysis of varying PHB contents in wild-type cells. a Fluorescence microscopy of wild-type cells after 14 days of nitrogen starvation. PHB granules are visualized by staining with Nile red. b TEM picture of wild-type cells after 17 days of nitrogen starvation. Representative PHB granules are indicated in two different cells by red asterisks. Figure modified from Koch et al. [2020a].
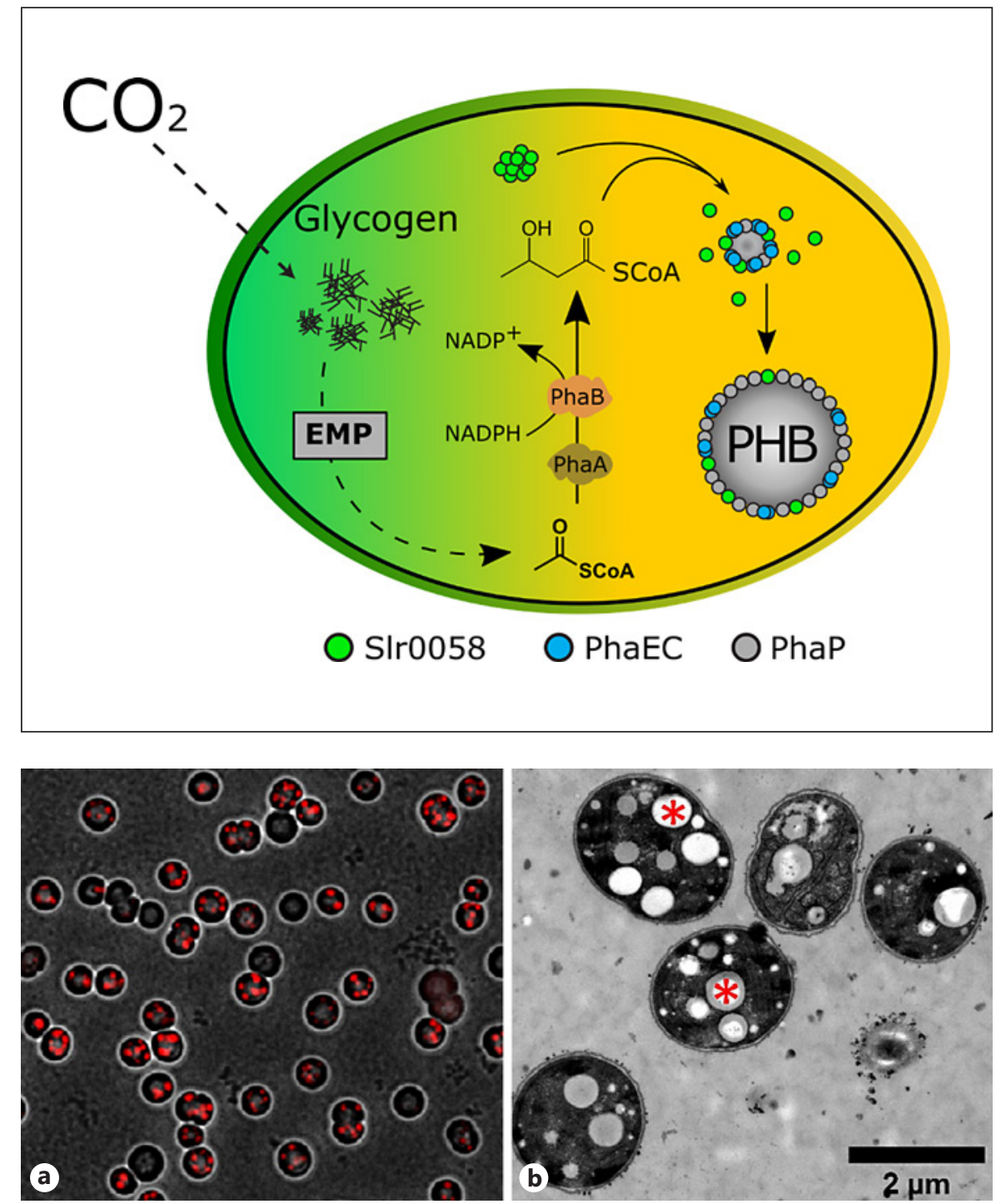

ules. Therefore, the surface area of those is higher compared to the fewer granules in the wild-type. Hence, it is likely that the $\Delta$ slr0058 mutant suffers from unintended interactions between the PHB surface and the cell's interior. Protein localization using fluorescent proteintagged Slr0058 showed that it aggregated in distinct foci during vegetative growth and these foci dispersed during the course of chlorosis. This led to the hypothesis that Slr0058 could act as an initial aggregation point for the PHB synthase PhaEC to direct the initiation of PHB synthesis and avoid uncontrolled formation of random $\mathrm{PHB}$ granules. Subsequently, it dissociates from maturating PHB granules.

Another protein involved in the regulation of number and size of PHB granules is PhaP (Ssl2501) [Hauf et al., 2015]. PhaP is directly located at the surface of PHB granules and hence considered a classical phasin. A PhaP deletion mutant showed half as many PHB granules as the wild-type. At the same time, the granules in the $\Delta$ phaP strain were larger than in the wild-type. The presence of PhaP and Slr0058 shows that the formation and maintenance of PHB granules in Synechocystis is tightly regulated. Decreased viability, for example in a $\Delta$ slr0058 mutant strain, underlines the importance of this regulation. A summary of the current understanding of the PHB metabolism in Synechocystis is shown in Figure 4.

Interestingly, in a nitrogen-starved Synechocystis population, the distribution of PHB granules among the cells is quite heterogenous (Fig. 5). Fluorescence microscopy 
showed that a large number of cells contained only smallto-medium amounts of PHB, while a few cells within the same population accumulated larger quantities [Koch et al., 2020a]. This indicates that the formation of PHB could be a bet-hedging strategy of the cells to be prepared for different future conditions [Ratcliff and Denison, 2010]. This highlights that the ability to possess PHB might be relevant under only very specific scenarios. The fact that so many cyanobacterial species produce $\mathrm{PHB}$ and formation of the granules is controlled in such a complex manner indicates that PHB fulfils an important physiological function and provides an evolutionary advantage to cyanobacteria in natural environments. These conditions could include, for example, the complex interactions with other microbes, which are difficult to mimic under laboratory conditions.

\section{A First Cyanobacterial Superproducer Strain}

Due to its industrial relevance as a bioplastic, considerable efforts have been made in the past years to increase the intracellular PHB concentrations in cyanobacteria, mainly by using genetic engineering. Several recent reviews cover this topic [Costa et al., 2018; Markl et al., 2018]. Cyanobacteria could in principle solve several drawbacks of PHB production with heterotrophic bacteria, such as avoiding the use of agricultural feedstock that is usually used for nutrition. However, the attempts published so far showed limited success in obtaining a high yield and production rates that would allow cost-efficient PHB production in a sustainable process. Very recently, the discovery of PirC, a central carbon flow regulator that controls the flux of carbon towards $\mathrm{PHB}$, now provides a new approach towards economically feasible PHB production in cyanobacteria. PirC is a small protein controlled by the PII signaling protein that exerts control over the activity of phosphoglycerate mutase [Orthwein et al., 2020]. Thereby, PirC directs the flow of carbon depending on the nitrogen availability. Mutant strains of PirC show an increased flux of newly fixed carbon towards pyruvate through lower glycolysis, thereby providing the building blocks for enhanced PHB synthesis. In combination with the overexpression of heterologous phaAB genes, the mutant strain PPT1 was created [Koch et al., 2020b]. Under phototrophic conditions, this strain accumulated $63 \%$ PHB per cell dry weight. Upon the addition of $10 \mathrm{~mm}$ acetate, PHB contents of more than $80 \%$ were reached, an amount that was so far only achieved by some heterotrophic bacteria. This demonstrates the potential of Synechocystis serving as a host for the sustainable production of industrial products.

\section{Conclusion and Outlook}

Despite the simple chemical structure of PHB, its granules are highly complex pseudo-organelles, with various different proteins involved in maintaining the correct function. Depending on the organism, PHB can serve numerous roles, from molecule storage to stress resistance and many more. Although the physiological function in cyanobacteria remains puzzling, the field of research is constantly expanding, thereby gaining deeper insights into its role. One of the most promising hypotheses is the role of PHB for the regulation of the intracellular redox balance. As an implication for biotechnological approaches this implies that when increased amounts of PHB are desired, cultivation conditions which favor high levels of reduction equivalents should be considered. Still, fundamental questions remain, such as a yet undiscovered PHB depolymerase. Since cyanobacteria grow photoautotrophically, they could serve as a chassis for a carbon-neutral, sustainable production of PHB. Further research in this field will hence be not only beneficial for basic research, but may also provide knowledge of industrial relevance.

\section{Conflict of Interest Statement}

The authors have no conflicts of interest to declare.

\section{Funding Sources}

Work in the Forchhammer lab was funded by the RTG 1708 "Molecular principles of bacterial survival strategies" and a scholarship to M.K. by the Studienstiftung des deutschen Volkes. We further acknowledge support by the Open Access Publishing Fund of the University of Tübingen.

\section{Author Contributions}

Conceptualization: K.F. Writing - original draft preparation: M.K. Review and editing: M.K. and K.F. 


\section{References}

Anderson AJ, Dawes EA. Occurrence, metabolism, metabolic role, and industrial uses of bacterial polyhydroxyalkanoates. Microbiol Rev. 1990; 54(4):450-72.

Arias S, Bassas-Galia M, Molinari G, Timmis KN Tight coupling of polymerization and depolymerization of polyhydroxyalkanoates ensures efficient management of carbon resources in Pseudomonas putida. Microb Biotechnol. 2013;6(5):551-63.

Arora NK, Singhal V, Maheshwari DK. Salinity-induced accumulation of poly- $\beta$-hydroxybutyrate in rhizobia indicating its role in cell protection. World J Microbiol Biotechnol. 2006;22(6):6036.

Batista MB, Teixeira CS, Sfeir MZT, Alves LPS, Valdameri G, Pedrosa FO, et al . PHB biosynthesis counteracts redox stress in Herbaspirillum seropedicae. Front Microbiol. 2018;9:472.

Beck C, Knoop H, Axmann IM, Steuer R. The diversity of cyanobacterial metabolism: genome analysis of multiple phototrophic microorganisms. BMC Genomics. 2012;13:56-

Bresan S, Sznajder A, Hauf W, Forchhammer K, Pfeiffer D, Jendrossek D. Polyhydroxyalkanoate (PHA) granules have no phospholipids. Sci Rep. 2016;6:26612.

Cassuriaga APA, Freitas BCB, Morais MG, Costa JAV. Innovative polyhydroxybutyrate production by Chlorella fusca grown with pentoses. Bioresour Technol. 2018;265:456-63.

Castro-Sowinski S, Burdman S, Matan O, Okon Y. Natural functions of bacterial polyhydroxyalkanoates. In: Chen GQ, editor. Plastics from bacteria: natural functions and applications. Microbiology Monographs. Berlin: Springer; 2009.

Chen X, Schreiber K, Appel J, Makowka A, Fähnrich B, Roettger M, et al. The Entner-Doudoroff pathway is an overlooked glycolytic route in cyanobacteria and plants. Proc Natl Acad Sci USA. 2016;113(19):5441.

Costa JAV, Moreira JB, Lucas BF, Braga VS, Cassuriaga APA, Morais MG. Recent advances and future perspectives of $\mathrm{PHB}$ production by cyanobacteria. Ind Biotechnol. 2018;14(5):249-56.

Damrow R, Maldener I, Zilliges Y. The multiple functions of common microbial carbon polymers, glycogen and $\mathrm{PHB}$, during stress responses in the non-diazotrophic cyanobacterium Synechocystis sp. PCC 6803. Front Microbiol. 2016;7:966.

De Eugenio LI, Escapa IF, Morales V, Dinjaski N, Galán B, García JL, et al. The turnover of medium-chain-length polyhydroxyalkanoates in Pseudomonas putida KT2442 and the fundamental role of PhaZ depolymerase for the metabolic balance. Environ Microbiol. 2010;12(1) 207-21.

De Philippis R, Ena A, Guastiini M, Sili C, Vincenzini M. Factors affecting poly- $\beta$ hydroxybutyrate accumulation in cyanobacteria and in purple non-sulfur bacteria. FEMS Microbiol Lett. 1992;103(2-4):187-94.
De Smet MJ, Eggink G, Witholt B, Kingma J, Wynberg $\mathrm{H}$. Characterization of intracellular inclusions formed by Pseudomonas oleovorans during growth on octane. J Bacteriol. 1983;154(2): 870-8

Doello S, Klotz A, Makowka A, Gutekunst K, Forchhammer K. A specific glycogen mobilization strategy enables rapid awakening of dormant cyanobacteria from chlorosis. Plant Physiol. 2018;177(2):594-603.

Eggers J, Steinbüchel A. Poly(3-hydroxybutyrate) degradation in Ralstonia eutropha H16 is mediated stereoselectively to (S)-3-hydroxybutyryl coenzyme A (CoA) via crotonyl-CoA. J Bacteriol. 2013;195(14):3213-23.

Eggers J, Steinbüchel A. Impact of Ralstonia eutropha's poly(3-Hydroxybutyrate) (PHB) depolymerases and phasins on $\mathrm{PHB}$ storage in recombinant Escherichia coli. Appl Environ Microbiol. 2014;80(24):7702-9.

Griebel R, Smith Z, Merrick JM. Metabolism of poly( $\beta$-hydroxybutyrate). I. Purification, composition, and properties of native $\operatorname{poly}(\beta$ hydroxybutyrate) granules from Bacillus megaterium. Biochemistry. 1968;7:3676-81.

Grundel M, Scheunemann R, Lockau W, Zilliges Y. Impaired glycogen synthesis causes metabolic overflow reactions and affects stress responses in the cyanobacterium Synechocystis sp. PCC 6803. Microbiology. 2012;158:3032-43.

Hauf W, Schlebusch M, Hüge J, Kopka J, Hagemann M, Forchhammer K. Metabolic changes in Synechocystis PCC6803 upon nitrogen-starvation: excess NADPH sustains polyhydroxybutyrate accumulation. Metabolites. 2013;3(1): 101-18.

Hauf W, Watzer B, Roos N, Klotz A, Forchhammer K. Photoautotrophic polyhydroxybutyrate granule formation is regulated by cyanobacterial phasin PhaP in Synechocystis sp. strain PCC 6803. Appl Environ Microbiol. 2015; 81(13):4411-22.

Haywood GW, Anderson AJ, Williams DR, Dawes EA, Ewing DF. Accumulation of a Poly(hydroxyalkanoate) copolymer containing primarily 3-hydroxyvalerate from simple carbohydrate substrates by Rhodococcus sp. NCIMB 40126. Int J Biol Macromol. 1991; 13(2):83-8.

Hein S, Tran H, Steinbüchel A. Synechocystis sp. PCC6803 possesses a two-component polyhydroxyalkanoic acid synthase similar to that of anoxygenic purple sulfur bacteria. Arch Microbiol. 1998;170(3):162-70.

Jendrossek D. Polyhydroxyalkanoate granules are complex subcellular organelles (carbonosomes). J Bacteriol. 2009;191(10):3195-202.

Jendrossek D, Handrick R. Microbial degradation of polyhydroxyalkanoates. Annu Rev Microbiol. 2002;56:403-32.

Jendrossek D, Pfeiffer D. New insights in the formation of polyhydroxyalkanoate granules (carbonosomes) and novel functions of poly(3-hydroxybutyrate). Environ Microbiol. 2014; 16(8):2357-73.
Jones LB, Ghosh P, Lee JH, Chou CN, Kunz DA. Linkage of the Nit1C gene cluster to bacterial cyanide assimilation as a nitrogen source. $\mathrm{Mi}$ crobiology. 2018;164(7):956-68.

Kadouri D, Burdman S, Jurkevitch E, Okon Y. Identification and isolation of genes involved in poly(beta-hydroxybutyrate) biosynthesis in Azospirillum brasilense and characterization of a phbC mutant. Appl Environ Microbiol. 2002; 68(6):2943-9.

Kadouri D, Jurkevitch E, Okon Y. Involvement of the reserve material poly-beta-hydroxybutyrate in Azospirillum brasilense stress endurance and root colonization. Appl Environ Microbiol. 2003a;69(6):3244-50.

Kadouri D, Jurkevitch E, Okon Y. Poly $\beta$-hydroxybutyrate depolymerase (PhaZ) in Azospirillum brasilense and characterization of a phaZ mutant. Arch Microbiol. 2003b;180: 309-18.

Kaewbai-Ngam A, Incharoensakdi A, Monshupanee $T$. Increased accumulation of polyhydroxybutyrate in divergent cyanobacteria under nutrient-deprived photoautotrophy: an efficient conversion of solar energy and carbon dioxide to polyhydroxybutyrate by Calothrix scytonemicola TISTR 8095. Bioresour Technol. 2016;212:342-7.

Kalia VC, Lal S, Cheema S. Insight in to the phylogeny of polyhydroxyalkanoate biosynthesis: horizontal gene transfer. Gene. 2007;389(1): 19-26.

Kavitha G, Kurinjimalar C, Sivakumar K, Palani P, Rengasamy R. Biosynthesis, purification and characterization of polyhydroxybutyrate from Botryococcus braunii kütz. Int J Biol Macromol. 2016;89:700-6.

Klotz A, Forchhammer K. Glycogen, a major player for bacterial survival and awakening from dormancy. Future Microbiol. 2017;12:101.

Klotz A, Georg J, Bučinská L, Watanabe S, Reimann $\mathrm{V}$, Januszewski W, et al . Awakening of a dormant cyanobacterium: resuscitation of chlorotic cells reveals a genetically determined program. Curr Biol. 2016;26(21):2862-72.

Köbler C, Schultz S-J, Kopp D, Voigt K, Wilde A. The role of the Synechocystis sp. PCC 6803 homolog of the circadian clock output regulator RpaA in day-night transitions. Mol Microbiol. 2018;110:847-61.

Koch M, Berendzen KW, Forchhammer AK. On the role and production of polyhydroxybutyrate (PHB) in the cyanobacterium Synechocystis sp. PCC 6803. Life.2020a;10(4):47.

Koch M, Bruckmoser J, Scholl J, Hauf W, Rieger B, Forchhammer K. Maximizing PHB content in Synechocystis sp. PCC 6803: development of a new photosynthetic overproduction strain. bioRxiv.2020b.doi:10.1101/2020.10.22.350660.

Koch M, Doello S, Gutekunst K, Forchhammer K. $\mathrm{PHB}$ is produced from glycogen turn-over during nitrogen starvation in Synechocystis sp. PCC 6803. IJMS. 2019;20(8):1942. 
Koch M, Forchhammer K. Storage polymers in cyanobacteria: friend or foe? In: Hudson P, editor. Cyanobacteria biotechnology. Hoboken: Wiley; 2020.

Koch M, Orthwein T, Alford JT, Forchhammer K. The Slr0058 protein from Synechocystis sp. PCC 6803 is a novel regulatory protein involved in PHB granule formation. Front Microbiol. 2020c;11:809-.

Koskimäki JJ, Kajula M, Hokkanen J, Ihantola E-L, Kim JH, Hautajärvi H, et al . Methyl-esterified 3-hydroxybutyrate oligomers protect bacteria from hydroxyl radicals. Nat Chem Biol. 2016; 12:332-8.

Kucho K, Okamoto K, Tsuchiya Y, Nomura S, Nango M, Kanehisa M, et al. Global analysis of circadian expression in the cyanobacterium Synechocystis sp. strain PCC 6803. J Bacteriol. 2005;187(6):2190-9.

Lemoigne M. Produit de déshydratation et de polymérisation de l'acide b-oxybutyrique. Bull Soc Chim Biol. 1926;8:770-82.

Liu G, Cai S, Hou J, Zhao D, Han J, Zhou J, et al . Enoyl-CoA hydratase mediates polyhydroxyalkanoate mobilization in Haloferax mediterranei. Sci Rep. 2016;6:24015.

Markl E, Grünbichler H, Lackner M. Cyanobacteria for PHB bioplastics production: a review. In: Wong YK, editor. Algae. Intech Open; 2018.

Martínez V, Jurkevitch E, García JL, Prieto MA. Reward for Bdellovibrio bacteriovorus for preying on a polyhydroxyalkanoate producer. Environ Microbiol. 2013;15:1204-15.

Mezzina MP, Pettinari MJ. Phasins, multifaceted polyhydroxyalkanoate granule-associated proteins. Appl Environ Microbiol. 2016;82(17): 5060-7.

Müller-Santos M, Koskimäki JJ, Alves LPS, De Souza EM, Jendrossek D, Pirttilä AM. The protective role of $\mathrm{PHB}$ and its degradation products against stress situations in bacteria. FEMS Microbiol Rev. 2020. doi: 10.1093/femsre/fuaa058.

Nowroth V, Marquart L, Jendrossek D. Low temperature-induced viable but not culturable state of Ralstonia eutropha and its relationship to accumulated polyhydroxybutyrate. FEMS Microbiol Lett. 2016:363(23):fnw249.

Obruca S, Sedlacek P, Slaninova E, Fritz I, Daffert $\mathrm{C}$, Meixner K, et al . Novel unexpected functions of PHA granules. Appl Microbiol Biotechnol. 2020;104(11):4795-810.

Orthwein T, Scholl J, Spät P, Lucius S, Koch M, Macek B, et al . The novel PII-Interacting regulator PirC (Sll0944) identifies 3-phosphoglycerate mutase (PGAM) as central control point of carbon storage metabolism in cyanobacteria. bioRxiv. 2020. doi: 10.1101/2020.09.11.292599.

Panda B, Jain P, Sharma L, Mallick N. Optimization of cultural and nutritional conditions for accumulation of poly-beta-hydroxybutyrate in Synechocystis sp. PCC 6803. Bioresour Technol. 2006;97(11):1296-301.

Pfeiffer D, Jendrossek D. Localization of poly(3-hydroxybutyrate) ( $\mathrm{PHB}$ ) granule-associated proteins during $\mathrm{PHB}$ granule formation and identification of two new phasins, PhaP6 and
PhaP7, in Ralstonia eutropha H16. J Bacteriol. 2012;194(21):5909-21.

Pfeiffer D, Jendrossek D. PhaM is the physiological activator of poly(3-hydroxybutyrate) (PHB) synthase (PhaC1) in Ralstonia eutropha. Appl Environ Microbiol. 2014;80(2):555-63.

Pohlmann A, Fricke WF, Reinecke F, Kusian B, Liesegang $\mathrm{H}$, Cramm R, et al . Genome sequence of the bioplastic-producing "Knallgas" bacterium Ralstonia eutropha H16. Nat Biotechnol. 2006;24(10):1257-62.

Pötter M, Madkour MH, Mayer F, Steinbüchel A. Regulation of phasin expression and polyhydroxyalkanoate (PHA) granule formation in Ralstonia eutropha H16. Microbiology. 2002; 148:2413-26.

Pötter M, Müller H, Reinecke F, Wieczorek R, Fricke F, Bowien B, et al . The complex structure of polyhydroxybutyrate (PHB) granules: four orthologous and paralogous phasins occur in Ralstonia eutropha. Microbiology. 2004;150: 2301-11.

Prieto MA, Bühler B, Jung K, Witholt B, Kessler B. $\mathrm{PhaF}$, a polyhydroxyalkanoate-granule-associated protein of Pseudomonas oleovorans GPol involved in the regulatory expression system for pha genes. J Bacteriol. 1999;181(3):858-68.

Prieto A, Escapa IF, Martínez V, Dinjaski N, Herencias C, de la Peña F, et al . A holistic view of polyhydroxyalkanoate metabolism in Pseudomonas putida. Environ Microbiol. 2016;18(2): $341-57$.

Raberg M, Voigt B, Hecker M, Steinbüchel A. A closer look on the polyhydroxybutyrate- (PHB-) negative phenotype of Ralstonia eutropha PHB-4. PLoS One. 2014;9(5):e95907.

Ratcliff WC, Denison RF. Individual-level bet hedging in the bacterium Sinorhizobium meliloti. Curr Biol. 2010;20(19):1740-4.

Reddy CS, Ghai R, Rashmi, Kalia VC. Polyhydroxyalkanoates: an overview. Bioresour Technol. 2003;87(2):137-46.

Ren Q, De Roo G, Ruth K, Witholt B, Zinn M, Thöny-Meyer L. Simultaneous accumulation and degradation of polyhydroxyalkanoates: futile cycle or clever regulation? Biomacromolecules. 2009;10(4):916-22.

Ruiz JA, López NI, Méndez BS. rpoS gene expression in carbon-starved cultures of the polyhydroxyalkanoate-accumulating species Pseudomonas oleovorans. Curr Microbiol. 2004;48(6): 396-400.

Saha R, Liu D, Hoynes-O'connor A, Liberton M, Yu J, Bhattacharyya-Pakrasi M, et al . Diurnal regulation of cellular processes in the cyanobacterium Synechocystis sp. strain PCC 6803: in sights from transcriptomic, fluxomic, and physiological analyses. mBio. 2016;7(3).

Schembri MA, Woods AA, Bayly RC, Davies JK. Identification of a $13-\mathrm{kDa}$ protein associated with the polyhydroxyalkanoic acid granules from Acinetobacter spp. FEMS Microbiol Lett. 1995;133(3):277-83.

Schlebusch M, Forchhammer K. Requirement of the nitrogen starvation-induced protein S110783 for polyhydroxybutyrate accumulation in Synechocystis sp. strain PCC 6803. Appl Environ Microbiol. 2010;76(18):6101-7.

Stal L, Moezelaar R. Fermentation in cyanobacteria. FEMS Microbiol Rev. 1997;21(2):179-211.

Timm A, Steinbüchel A. Formation of polyesters consisting of medium-chain-length 3-hydroxyalkanoic acids from gluconate by Pseudomonas aeruginosa and other fluorescent pseudomonads. Appl Environ Microbiol. 1990; 56(11):3360-7.

Valappil SP, Misra SK, Boccaccini AR, Keshavarz T, Bucke C, Roy I. Large-scale production and efficient recovery of PHB with desirable material properties, from the newly characterised Bacillus cereus SPV. J Biotechnol. 2007;132(3): 251-8.

Van der Walle GA, De Koning GJ, Weusthuis RA, Eggink G. Properties, modifications and applications of biopolyesters. Adv Biochem Eng Biotechnol. 2001;71:263-91.

Van Gemerden H. On the ATP generation by Chromatium in darkness. Arch Mikrobiol. 1968;64(2):118-24.

Wang C, Sheng X, Equi RC, Trainer MA, Charles TC, Sobral BW. Influence of the poly-3-hydroxybutyrate $(\mathrm{PHB})$ granule-associated proteins (PhaP1 and PhaP2) on PHB accumulation and symbiotic nitrogen fixation in Sinorhizobium meliloti Rm1021. J Bacteriol. 2008; 189(24):9050-6.

Watzer B, Forchhammer K. Cyanophycin synthesis optimizes nitrogen utilization in the unicellular cyanobacterium Synechocystis sp. strain PCC 6803. Appl Environ Microbiol. 2018;84(20): e01298-18.

Yabuuchi E, Kosako Y, Yano I, Hotta H, Nishiuchi Y. Transfer of two Burkholderia and an Alcaligenes species to Ralstonia gen. Nov.: proposal of Ralstonia pickettii (Ralston, Palleroni and Doudoroff 1973) comb. Nov., Ralstonia solanacearum (Smith 1896) comb. Nov. and Ralstonia eutropha (Davis 1969) comb. Nov. Microbiol Immunol. 1995;39(11):897-904.

York GM, Stubbe J, Sinskey AJ. New insight into the role of the PhaP phasin of Ralstonia eutropha in promoting synthesis of polyhydroxybutyrate. J Bacteriol. 2001;183(7):2394-7.

York GM, Stubbe J, Sinskey AJ. The Ralstonia eutropha PhaR protein couples synthesis of the PhaP phasin to the presence of polyhydroxybutyrate in cells and promotes polyhydroxybutyrate production. J Bacteriol. 2002;184(1):59-66.

Zhang H, Liu Y, Yao C, Cao X, Tian J, Xue S. FabG can function as $\mathrm{PhaB}$ for poly-3-hydroxybutyrate biosynthesis in photosynthetic cyanobacteria Synechocystis sp. PCC 6803. Bioengineered. 2017;8:1-9.

Zhang X, Lin Y, Wu Q, Wang Y, Chen GQ. Synthetic biology and genome-editing tools for improving PHA metabolic engineering. Trends Biotechnol. 2020;38(7):689-700.

Zhao YH, Li HM, Qin LF, Wang HH, Chen GQ. Disruption of the polyhydroxyalkanoate synthase gene in Aeromonas hydrophila reduces its survival ability under stress conditions. FEMS Microbiol Lett. 2007;276(1):34-41. 